\title{
Initial Sequential Organ Failure Assessment score versus Simplified Acute Physiology score to analyze multiple organ dysfunction in infectious diseases in Intensive Care Unit
}

\author{
Remyasri Nair, Nithish M. Bhandary, Ashton D. D'Souza ${ }^{1}$
}

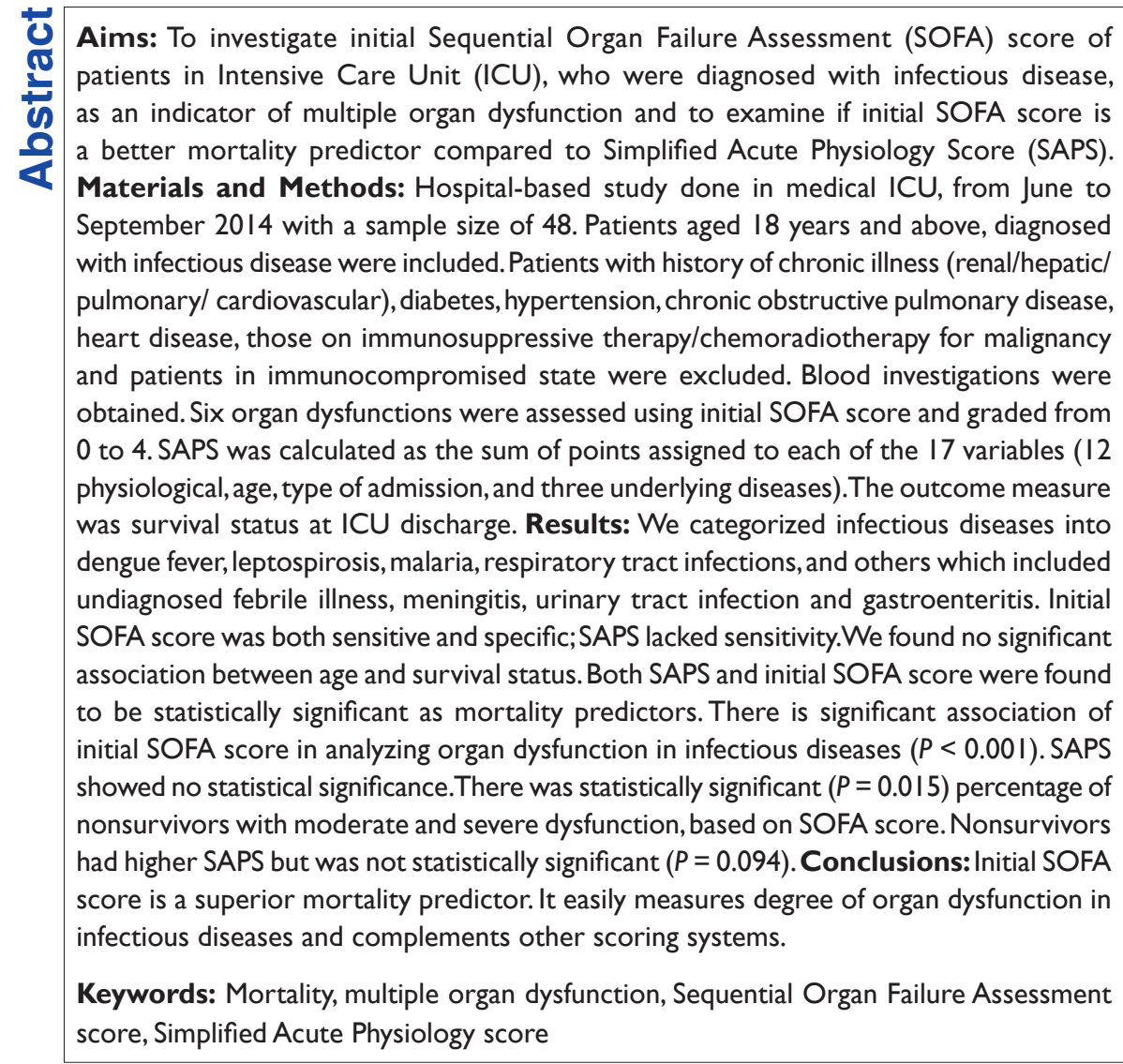

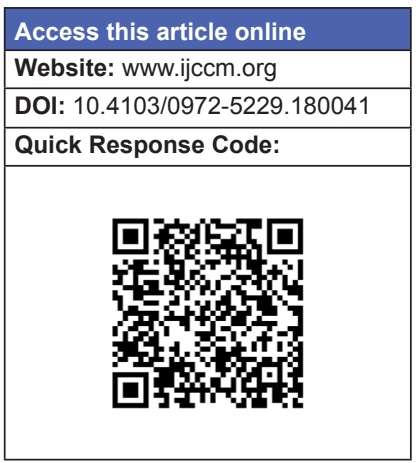

From:

Departments of General Medicine and ${ }^{1}$ Anesthesia, A. J. Institute of Medical Sciences, Mangalore, Karnataka, India

\section{Correspondence:}

Dr. Remyasri Nair, Room Number: 203, PG Residents Hostel, A. J. Institute of Medical Sciences, Kuntikana, Mangalore - 575 004, Karnataka, India. E-mail: remyasri.15@gmail.com
This is an open access article distributed under the terms of the Creative Commons Attribution-NonCommercial-ShareAlike 3.0 License, which allows others to remix, tweak, and build upon the work non-commercially, as long as the author is credited and the new creations are licensed under the identical terms.

For reprints contact: reprints@medknow.com

How to cite this article: Nair R, Bhandary NM, D'Souza AD. Initial Sequential Organ Failure Assessment score versus Simplified Acute Physiology score to analyze multiple organ dysfunction in infectious diseases in Intensive Care Unit. Indian J Crit Care Med 2016;20:210-5. 


\section{Introduction}

Infectious diseases cause multiple organ dysfunctions (MODs). Lack of clinical diagnostic criteria, high cost, and nonavailability of isolation techniques result in misdiagnosis, treatment failure, and improper utilization of resources. ${ }^{[1]}$ Etiology remains unestablished during the first crucial 24-48 h. The European Society of Intensive Care Medicine organized a consensus meeting in Paris in October 1994 to create a so-called Sepsis-related Organ Failure Assessment (SOFA) score, ${ }^{[1]}$ to describe quantitatively and as objectively as possible the degree of organ dysfunction over time in groups of patients or even in individual patients. Six organ dysfunctions were assessed using initial SOFA score and graded from 0 to 4 . There are two major applications of such a SOFA score: To improve our understanding of the natural history of organ dysfunction and to assess the effects of new therapies on the course of organ dysfunction. This could be used to characterize patients at entry or to evaluate the effects of treatment. ${ }^{[1]}$ Simplified Acute Physiology Score (SAPS) was introduced in France in 1984 which included 13 variables and age. Later in 1993, it was modified to SAPS $\mathrm{II}^{[2]}$ which was calculated as the sum of points assigned to each of the 17 variables (12 physiological variables, age, type of admission, and three underlying diseases). Previously used scores such as SAPS, Acute Physiology Age Chronic Health Evaluation (APACHE) ${ }^{[3]}$ did not focus on the severity of MOD. They could only estimate prognosis and compare results among Intensive Care Units (ICU's). The physiological parameters in these scores were affected by treatment. These scores did not guide decisions regarding therapeutic interventions. Serial evaluation of therapy-related improvement could not be done using these scores. The evaluation of organ dysfunction should be based on a limited number of simple but objective variables that are easily and routinely measured in every institution. The collection of this information should not impose any intervention beyond what is routinely performed in every ICU. The variables used should as much as possible be independent of therapy since therapeutic management may vary from one institution to another and even from one patient to another. According to the Medline search, no study comparing these two scoring systems had been reported in our region (Southeast Asia). Hence, we designed a study to compare two scoring systems SAPS and SOFA score in ICU, which may be helpful in prognosticating the severity of organ dysfunction and predicting mortality in infectious diseases in Indian population.

\section{Materials and Methods}

\section{Setting}

The study was conducted in a 900 bedded tertiary care hospital with 19 bedded Medical ICU. Physicians were primarily responsible for the patient care. However, treatment decisions were often made after discussions with the ICU team. The ICU team included an ICU consultant, shift duty doctors and nursing staff with average nurse to patient ratio 1:3.

\section{Data collection}

This study was done in medical ICU, from June 2014 to September 2014 (4 months) with a significant sample size of 48 patients. Patients aged 18 years and above, diagnosed with infectious disease were included in the study.

Patients with history of chronic illness (renal/hepatic/ pulmonary/cardiac), history of diabetes, hypertension, chronic obstructive pulmonary disease, and heart disease, those who were on immunosuppressive therapy/chemoradiotherapy for malignancy and those patients in immunocompromised state were excluded as these alter the parameters required for calculation of both the scores. The study was approved by the Ethical Committee of our university. Patients data observed during the first $24 \mathrm{~h}$ of their hospital stay were collected: Demographics, temperature $\left({ }^{\circ} \mathrm{C}\right)$, systolic and mean arterial blood pressure $(\mathrm{mmHg})$, heart rate, respiratory rate, partial pressure of oxygen $(\mathrm{mmHg})$ and fraction of inspired oxygen, arterial $\mathrm{pH}$ and bicarbonate, serum sodium, potassium, urea and creatinine, urine output, serum white blood cell count, hematocrit, platelet count and bilirubin, age, type of admission, Glasgow Coma Scale (GCS), presence of chronic diseases (chronic organ insufficiency) or immunocompromised state. Assessment of the severity using GCS is problematic when ongoing sedation is needed. Hence, the lowest initial recording of GCS before sedation was used.

Initial SOFA score and SAPS were calculated based on the worst values of the first $24 \mathrm{~h}$ of admission. All enrolled patients were followed during their ICU stay, and main outcome measure was survival status at ICU discharge.

Data were entered in Microsoft Excel Sheet, and statistical analysis was done using SPSS 11 (SPSS Inc., Chicago, Illinois, USA). Appropriate statistical tests such as Chi-square test and other nonparametric tests were used. $P<0.05$ was considered statistically significant. 
Discrimination is how well a model can predict outcome, is tested by calculating area under receiver operating characteristics (ROC) curve, a graphical plot of true positive (sensitivity) against false positive rate (1-specificity) and the best cutoff value was derived.

\section{Results}

In our study, we categorized infectious diseases into dengue fever, leptospirosis, malaria, respiratory tract infections (RTIs) such as pneumonia, tuberculosis, bronchitis, Bronchiectasis, other lower RTI and others which included undiagnosed febrile illness, meningitis, urinary tract infection (UTI), and gastroenteritis. A majority of cases in this study, 44\%, were RTIs, followed by malaria which was $27 \%$, and other infections which included undiagnosed febrile illness, meningitis, UTI and gastroenteritis constituted $21 \%$ of the cases. Only $6 \%$ were cases of leptospirosis, and $2 \%$ were diagnosed as dengue.

Discrimination for initial SOFA score and SAPS were fair with area under ROC curve of 0.748 and 0.742 respectively [Figure 1]. The cutoff point for initial SOFA score was 9.5 and for SAPS was 61.

The gold standard was taken as survival status at ICU discharge. Based on this initial SOFA score was both sensitive and specific. However, SAPS lacked sensitivity [Table 1].

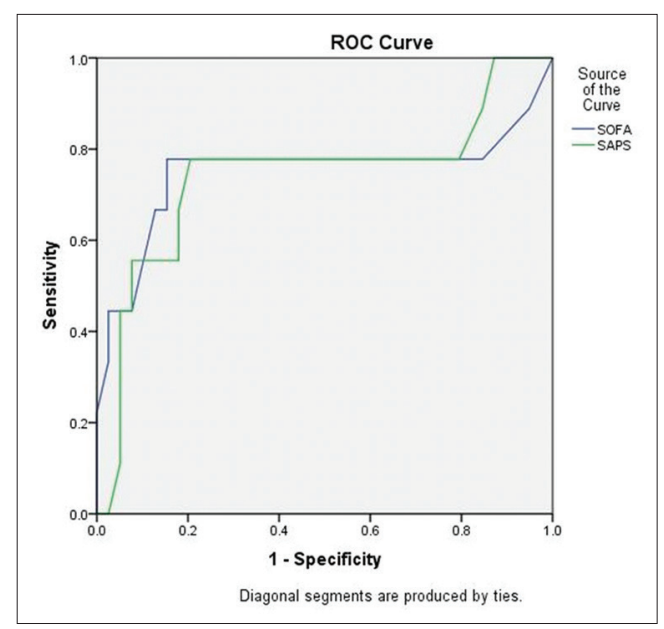

Figure I: Receiver operator characteristics curve for assessment of cutoff values
In our study, all the 48 patients met the inclusion and exclusion criteria. The mean age among nonsurvivors was 47.78 years and among survivors was 53.56 years. Of 48 patients, nine patients expired. We found no significant association between age and survival status $(P=0.43)$. In fact, survivors had a higher mean age compared to nonsurvivors. Independent $t$-test was used to compare SAPS and initial SOFA score as an indicator of survival status. Both SAPS and initial SOFA score were found to be statistically significant as mortality predictors [Table 2].

The mean initial SOFA score among nonsurvivors was 11.33 and among survivors was 6.46. The mean SAPS among nonsurvivors was 51.11 and among survivors was 34.54. Both mean initial SOFA score and SAPS were higher among nonsurvivors [Figure 2].

Mean initial SOFA score was highest among cases of malaria followed by leptospirosis. Whereas mean SAPS was highest among RTI followed by leptospirosis and malaria [Figure 3].

There is significant association of initial SOFA score in analyzing organ dysfunction in infectious diseases $(P<0.001)$.Whereas SAPS showed no statistical significance.

In our study, based on initial SOFA score, respiratory dysfunction was predominantly seen in RTI, followed by malaria and dengue. Coagulation was affected in malaria and Dengue more frequently followed by other infections (undiagnosed febrile illness, meningitis, UTI, and gastroenteritis) and leptospirosis in descending order. Hepatic involvement was predominantly seen in malaria

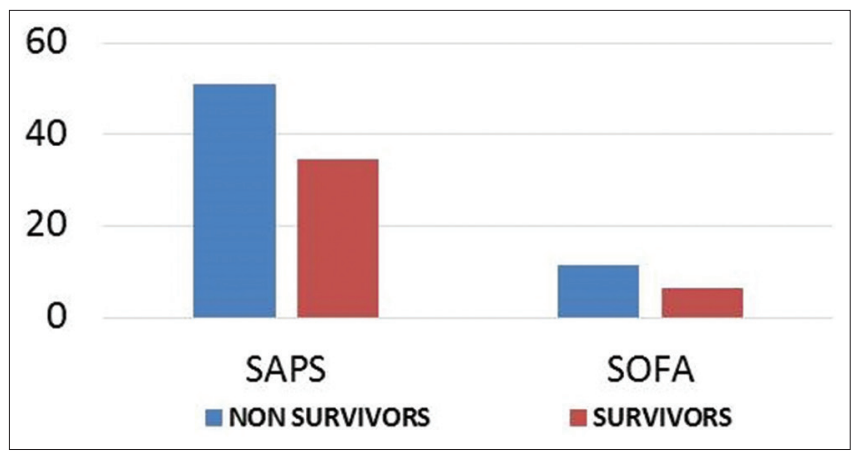

Figure 2: Comparison of mean initial Sequential Organ Failure Assessment score and Simplified Acute Physiology Score with survival status

Table I: Sensitivity and specificity of scores

\begin{tabular}{lccccccc}
\hline Parameter & $\begin{array}{c}\text { Sensitivity } \\
(\%)\end{array}$ & $\begin{array}{c}\text { Specificity } \\
(\%)\end{array}$ & $\begin{array}{c}\text { Positive predictive } \\
\text { value (\%) }\end{array}$ & $\begin{array}{c}\text { Negative predictive } \\
\text { value (\%) }\end{array}$ & $\begin{array}{c}\text { Diagnostic } \\
\text { accuracy (\%) }\end{array}$ & $\begin{array}{c}\text { Gold } \\
\text { standard }\end{array}$ & $\begin{array}{c}\text { Agreement } \\
(\%)\end{array}$ \\
\hline SOFA cutoff 9.5 & 77.80 & 84.60 & 53.80 & 94.30 & 83.33 & Survival & 83.33 \\
SAPS cutoff 61 & 44.40 & 94.90 & 66.70 & 88.10 & 85.42 & Survival & 85.42 \\
\hline
\end{tabular}

SOFA: Sequential Organ Failure Assessment, SAPS: Simplified Acute Physiology Score 
and other infections (undiagnosed febrile illness, meningitis, UTI and gastroenteritis) followed by RTI. This association was statistically significant $(P<0.001)$. In this study, cases of dengue had no hepatic involvement. Cardiovascular, central nervous system (CNS), and renal systems were also found to be affected in RTI, sparing coagulation. Cases of malaria, leptospirosis, dengue, and other infections (undiagnosed febrile illness, meningitis, UTI, and gastroenteritis) had significant MOD. Cardiovascular and renal dysfunctions were seen in all patients.

CNS involvement was seen in all except dengue. However, this association was not statistically significant.

Respiratory and renal dysfunctions were the most common among these infectious diseases. Chi-square test showed statistically significant association of respiratory, coagulatory and hepatic dysfunction in infectious diseases, based on initial SOFA score $(P<0.001)$.

Mild organ dysfunction was indicated by an initial SOFA score of $0-7$, the corresponding mortality rate was $4.2 \%$. A score of $8-15$ and $\geq 16$ suggested moderate and severe dysfunction respectively, with their mortality rates being $10.4 \%$ and $4.2 \%$ [Table 3]. Figure 4 shows a higher percent of nonsurvivors with moderate and severe organ dysfunction based on SOFA score. This was statistically significant by Chi-square test $(P=0.015)$.

We considered cases with SAPS of 0-25 to have mild risk of nonsurvival (mortality rate being $4.2 \%$ ), a score of $26-50$ to have moderate risk (mortality rate $-4.2 \%$ ) and scores of 51-76 and above to have severe risk of nonsurvival (mortality rate $-10.4 \%$ ) as shown in

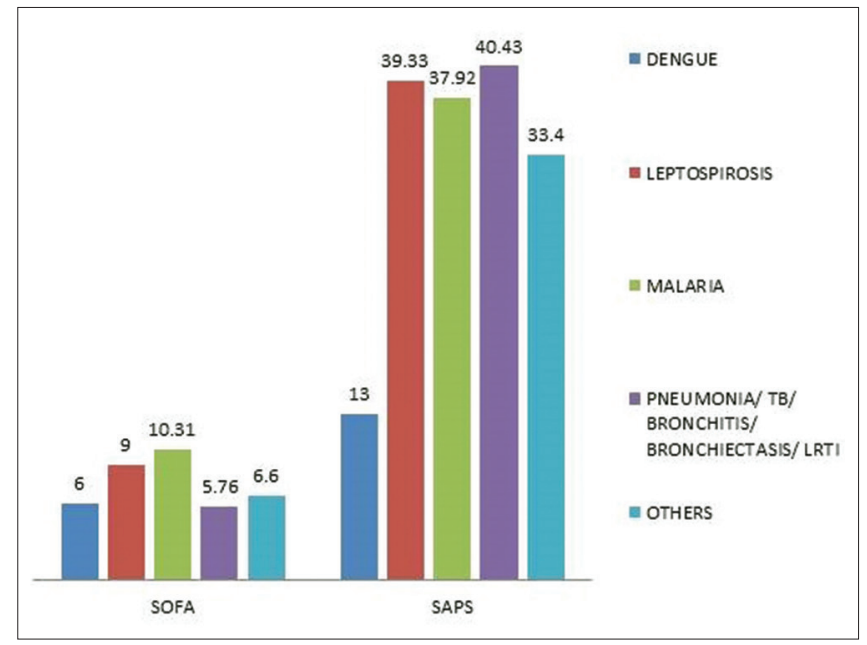

Figure 3: Mean Sequential Organ Failure Assessment and Simplified Acute Physiology score in infectious diseases
Table 4. Figure 5 shows higher percent of nonsurvivors with higher SAPS but was not statistically significant $(P=0.094)$.

\section{Discussion}

Both initial SOFA score and SAPS were significantly higher among nonsurvivors than survivors. Area under ROC curve observed for SOFA score model was 0.748 which was 0.772 as reported in a multicenter study outside India. ${ }^{[1]}$ Area under ROC curve observed in our study for SAPS model was 0.742 , which was 0.810 in a multicenter Austrian study. ${ }^{[2]}$ The discrimination of SOFA score model was better than SAPS model in our study, and the finding was consistent with Vincent et al. study. ${ }^{[1]}$

Initial SOFA score observed in our study ranged from 1 to 18 . Survivors had lower mean SOFA score compared with nonsurvivors which was statistically significant $(P=0.009)$ as observed in other studies. Mortality increased with increasing SOFA score, which was also statistically significant $(P=0.015)$. Similar results were found in other studies. ${ }^{[3,4]}$ Abhinandan and Vedavathi in 2013, conducted a study on usefulness of APACHE and SOFA score in analyzing patients with MOD in sepsis. The study concluded that serial measurement of

Table 2: Independent $t$-test for comparison of age, Simplified Acute Physiology score and Sequential Organ Failure Assessment with survival

\begin{tabular}{llcccccc}
\hline & Survival & $\boldsymbol{n}$ & Mean & SD & $\boldsymbol{t}$ & df & $\boldsymbol{P}$ value \\
\hline Age & Non survivors & 9 & 47.78 & 15.081 & -0.797 & 46 & 0.43 \\
& Survivors & 39 & 53.56 & 20.464 & & & \\
SAPS & Non survivors & 9 & 51.11 & 19.928 & 2.708 & 46 & 0.009 \\
& Survivors & 39 & 34.54 & 15.743 & & & \\
SOFA & Non survivors & 9 & 11.33 & 5.874 & 2.4 & 9.233 & 0.039 \\
& Survivors & 39 & 6.46 & 3.347 & & & \\
\hline
\end{tabular}

SD: Standard deviation, SOFA: Sequential Organ Failure Assessment, SAPS: Simplified Acute Physiology Score

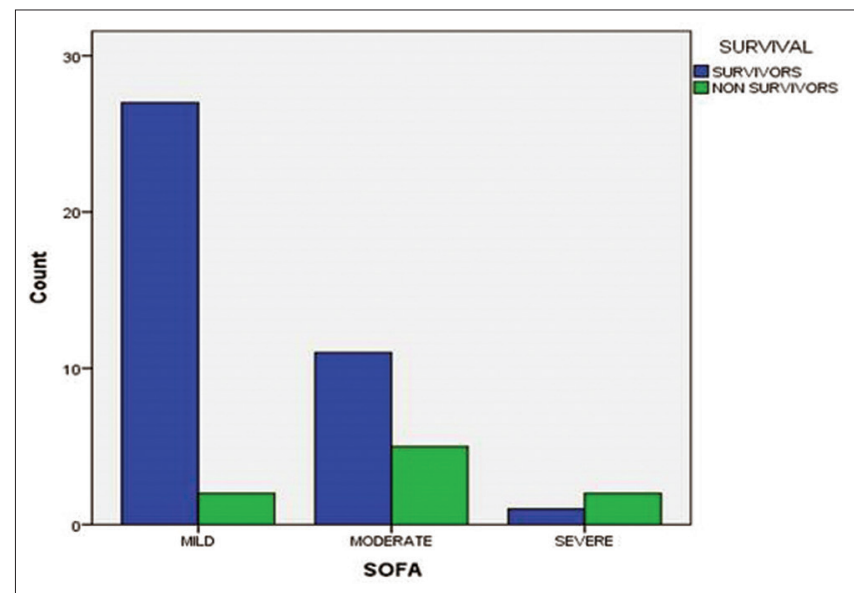

Figure 4: Initial Sequential Organ Failure Assessment score and survival status 


\begin{tabular}{|c|c|c|c|}
\hline & \multicolumn{2}{|c|}{ Survival } & \multirow[t]{2}{*}{ Total } \\
\hline & Survivors & Non survivors & \\
\hline \multicolumn{4}{|l|}{ SOFA } \\
\hline \multicolumn{4}{|l|}{ Mild } \\
\hline Count & 27 & 2 & 29 \\
\hline \% within survival & 69.2 & 22.2 & 60.4 \\
\hline \multicolumn{4}{|l|}{ Moderate } \\
\hline Count & II & 5 & 16 \\
\hline \% within survival & 28.2 & 55.6 & 33.3 \\
\hline \multicolumn{4}{|l|}{ Severe } \\
\hline Count & 1 & 2 & 3 \\
\hline$\%$ within survival & 2.6 & 22.2 & 6.2 \\
\hline \multicolumn{4}{|l|}{ Total } \\
\hline Count & 39 & 9 & 48 \\
\hline$\%$ within survival & 100.0 & 100.0 & 100.0 \\
\hline
\end{tabular}

\begin{tabular}{|c|c|c|c|}
\hline & \multicolumn{2}{|c|}{ Survival } & \multirow[t]{2}{*}{ Total } \\
\hline & Survivors & Non survivors & \\
\hline \multicolumn{4}{|l|}{ SAPS } \\
\hline \multicolumn{4}{|l|}{ Mild } \\
\hline Count & 12 & 2 & 14 \\
\hline$\%$ within survival & 30.8 & 22.2 & 29.2 \\
\hline \multicolumn{4}{|l|}{ Moderate } \\
\hline Count & 20 & 2 & 22 \\
\hline \% within survival & 51.3 & 22.2 & 45.8 \\
\hline \multicolumn{4}{|l|}{ Severe } \\
\hline Count & 7 & 5 & 12 \\
\hline$\%$ within survival & 17.9 & 55.6 & 25.0 \\
\hline \multicolumn{4}{|l|}{ Total } \\
\hline Count & 39 & 9 & 48 \\
\hline$\%$ within survival & 100.0 & 100.0 & 100.0 \\
\hline
\end{tabular}

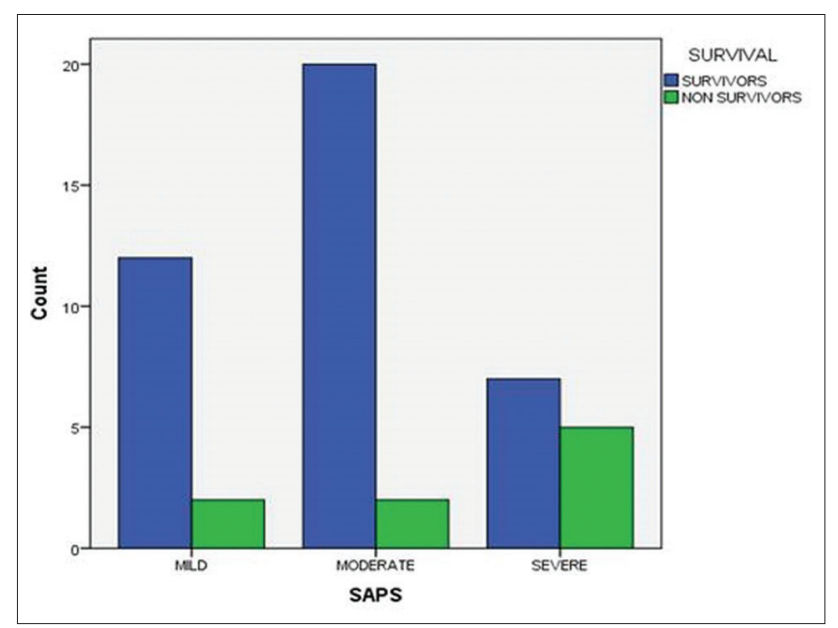

Figure 5: Simplified Acute Physiology score and survival status

SOFA score during the 1st week is a very useful tool in predicting the outcome. ${ }^{[3]}$ Our study proved significant association of initial SOFA score in analyzing organ dysfunction in infectious diseases $(P<0.001)$, similar to the study done in 1996, on SOFA score to describe organ dysfunction. A study in 2001 analyzed serial evaluation of SOFA score as an outcome predictor in critically ill and proved SOFA score during the first few days of ICU admission as a good indicator of prognosis. ${ }^{[4]}$

SAPS ranged from 6 to 76 in our study. Survivors had lower mean SAPS compared to nonsurvivors, which was statistically significant $(P=0.039)$. Mortality increased with increasing SAPS, which was not statistically significant $(P=0.094)$.

The bivariate analysis showed no statistically significant correlation of mortality with age group as observed in other studies.

SAPS showed no statistical significance in our study unlike in another study ${ }^{[5,6]}$ but similar to study done in Greek ICU by Katsaragakis et al. ${ }^{[7]}$ SOFA score model predicted mortality rate better than SAP scoring system in our ICU. The reason could probably be the consideration of presence or absence of mechanical ventilation, ionotropic support, and disease-specific subgroup analysis.

Few other studies combined SOFA score with APACHE II score to predict hospital mortality of critically ill medical and surgical patients and showed that combining both scores improved mortality prediction. ${ }^{[8,9]}$ Minne et al. in 2008 evaluated SOFA-based models for predicting mortality in ICU which suggested that SOFA-based models were comparable with APACHE II/III and were competitive with SAPS II in mortality prediction in medical/surgical ICU and another study proved APACHE II and SAPS as prognostic models in surgical ICU. ${ }^{[10,11]}$ Yet, another study compared APACHE III with initial SOFA score to predict ICU mortality and showed initial SOFA score is comparable to APACHE III score for mortality prediction in ICU. ${ }^{[12]}$

A study on SOFA score as prognostic marker in critically ill patients proved SOFA in ICU at presentation and at $48 \mathrm{~h}$ as a good indicator of prognosis ${ }^{[13]}$ which was similar to a study in Nepal by Acharya et al. ${ }^{[14]}$

There are no studies in India comparing SAPS with less known initial SOFA score in ICU, to analyze MOD in infectious diseases and to predict mortality. We moved a step further by even including only nonsurgical cases, a set of infectious diseases in ICU. This study showed initial SOFA score superior to SAPS not only as a mortality predictor but also as an indicator of MOD in infectious diseases in our ICU. We even derived the significance of each organ dysfunction based on initial SOFA score as a 
mortality predictor. Most common diseases causing MOD in our ICU were identified. We found that many cases were undiagnosed febrile illness without an etiology and SOFA score is an indicator of MOD and mortality in such cases. In such situations, it helps physicians to prioritize patients with regard to use of organ support and to modify therapeutic interventions.

More studies in multiple centers involving larger patient population are needed to validate both the scoring systems in developing countries like ours and separate scoring systems that factor in the pitfalls in resource-limited environment need to be developed for good predictability.

\section{Conclusion}

Mortality of patients was significantly high when initial SOFA score $\geq 11$ and SAPS $\geq 51$. Discrimination was fair for both models, but initial SOFA score was superior to SAPS. Initial SOFA score is a superior mortality predictor in infectious diseases in ICU. It helps physicians to prioritize patients with regard to use of organ support. Initial SOFA score is both sensitive and specific, hence, can be used as a better screening tool to analyze organ dysfunction. Initial SOFA score easily measures the degree of organ dysfunction in Infectious diseases, thereby helps physicians to modify therapeutic interventions. It will complement other scoring systems. SOFA score if measured daily monitors progression of the disease, which is more informative and improves accuracy. Large multicentric studies with large number of patients are needed to prove the same.

\section{Acknowledgment}

We would like to acknowledge the support of Dr. Sudhesh Rao (Chief Intensivist, Department of Critical Care) and Dr. Sreekanth (Statistician) to complete this study.

\section{Financial support and sponsorship} Nil.

\section{Conflicts of interest}

There are no conflicts of interest.

\section{References}

1. Vincent JL, Moreno R, Takala J, Willatts S, De Mendonça A, Bruining H, et al. The SOFA (Sepsis-related Organ Failure Assessment) score to describe organ dysfunction/failure. On behalf of the Working Group on Sepsis-Related Problems of the European Society of Intensive Care Medicine. Intensive Care Med 1996;22:707-10.

2. Metnitz PG, Valentin A, Vesely H, Alberti C, Lang T, Lenz K, et al. Prognostic performance and customization of the SAPS II: Results of a multicenter Austrian study. Simplified Acute Physiology Score. Intensive Care Med 1999;25:192-7.

3. Abhinandan KS, Vedavathi R. Usefulness of sequential organ failure assessment (SOFA) and acute physiology and chronic health evaluation II (APACHE II) score in analysing patients with multiple organ dysfunction syndrome in sepsis. J Evol Med Dent Sci 2013;2:9591-605.

4. Ferreira FL, Bota DP, Bross A, Mélot C, Vincent JL. Serial evaluation of the SOFA score to predict outcome in critically ill patients. JAMA 2001;286:1754-8.

5. Le Gall JR, Lemeshow S, Saulnier F. A new Simplified Acute Physiology Score (SAPS II) based on a European/North American multicenter study. JAMA 1993;270:2957-63.

6. Le Gall JR, Lemeshow S. The SAPS II: A new score with new objectives. In: Vincent JL, editor. Yearbook of Intensive Care and Emergency Medicine. Berlin: Springer Berlin; 1994. p. 795-804.

7. Katsaragakis S, Papadimitropoulos K, Antonakis P, Strergiopoulos S, Konstadoulakis MM, Androulakis G. Comparison of Acute Physiology and Chronic Health Evaluation II (APACHE II) and Simplified Acute Physiology Score II (SAPS II) scoring systems in a single Greek intensive care unit. Crit Care Med 62000;28:426-32.

8. Ho KM. Combining sequential organ failure assessment (SOFA) score with acute physiology and chronic health evaluation (APACHE) II score to predict hospital mortality of critically ill patients. Anaesth Intensive Care 2007;35:515-21.

9. Halim DA, Murni TW, Redjeki IS. Comparison of Apache II, SOFA, and modified SOFA scores in predicting mortality of surgical patients in intensive care unit at Dr. Hasan Sadikin General Hospital. Crit Care Shock 2009;12:157-69.

10. Minne L, Abu-Hanna A, de Jonge E. Evaluation of SOFA-based models for predicting mortality in the ICU: A systematic review. Crit Care 2008;12:R161.

11. Sakr Y, Krauss C, Amaral AC, Réa-Neto A, Specht M, Reinhart K, et al. Comparison of the performance of SAPS II, SAPS 3, APACHE II, and their customized prognostic models in a surgical intensive care unit. Br J Anaesth 2008;101:798-803.

12. Shrestha GS, Gurung R, Amatya R. Comparison of acute physiology, age, chronic health evaluation III score with initial sequential organ failure assessment score to predict ICU mortality. Nepal Med Coll J 2011;13:50-4.

13. Bale C, Kakrani AL, Dabadghao VS, Sharma ZD. Sequential organ failure assessment score as prognostic marker in critically ill patients in a tertiary care intensive care unit. Int J Med Public Health 2013;3:155.

14. Acharya SP, Pradhan B, Marhatta MN. Application of "the Sequential Organ Failure Assessment (SOFA) score" in predicting outcome in ICU patients with SIRS. Kathmandu Univ Med J (KUMJ) 2007;5:475-83. 